Errata

\title{
In Vitro Histological and Tetracycline Staining Properties of Surface Layer Rat Incisor Enamel also Reflect the Cyclical Nature of the Maturation Process
}

A. Boyde and E.J. Reith

Histochemistry (1982) 75:341-351

In the Materials and Methods, the data in parentheses at the end of the following paragraphs are being repeated in a clearer manner:

Short Term Wet Storage

(six 250-g animals)

Immediate Staining

(six 250-g animals)

Correlation with in Vivo and in Vitro Tetracycline Banding

a) (fifteen 270-g and nine 185-240 g animals)

b) (four $240-\mathrm{g}$ animals)

c) (four 240-g animals)

Perfusion Fixation

(six 100-g rats)

\section{Standardization of}

Formaldehyde-Induced Fluorescence and its Measurement

to Quantify Serotonin Emission in Pulmonary Neuroendocrine Cells

I.M. Keith, L.A. Wiley, and J.A. Will

Histochemistry (1982) 75:251-258

The inset (fluorescence micrograph) of the NEB in Figure 1 should be rotated through $180^{\circ}$, to match the phase contrast image of the same NEB. 